\title{
Terapia assistida por animais e sua influência nos níveis de pressão arterial de idosos institucionalizados
}

\section{Animal assisted therapy and its influence on blood pressure institutionalized elderly}

\author{
Fernanda de Toledo Vieira ${ }^{1}$, Racire Sampaio Silva ${ }^{2}$, Valéria Rosseto Lemos ${ }^{3}$, Romildo Rocha \\ Azevedo Júnior ${ }^{4}$, Marcel de Toledo Vieira ${ }^{5}$, Marcelo Renan de Deus Santos ${ }^{6}$, Guadalupe Gomes \\ Machado ${ }^{7}$, Daniele Vieira Baltar de Oliveira Jorge ${ }^{7}$, Irineu Vieira Lopes Neto ${ }^{7}$
}

Vieira FT, Silva RS, Lemos VR, Azevedo Júnior RR, Vieira MT, Santos MRD, Machado GG, Jorge DVBO, Lopes Neto IV. Terapia assistida por animais e sua influência nos níveis de pressão arterial de idosos institucionalizados / Animal assisted therapy and its influence on blood pressure institutionalized elderly. Rev Med (São Paulo). 2016 jul.-set.;95(3):122-7.

RESUMO: A terapia assistida por animais (TAA) é utilizada como instrumento no tratamento de doenças, com diversos efeitos benéficos em paciente psiquiátricos, hospitalizados, idosos, dentre outros. A TAA objetiva promover o bem-estar, saúde emocional, física, social e cognitiva, sendo o animal o principal agente da terapia, que funciona como ponte de ligação entre o tratamento e o paciente. A institucionalização do idoso ocorre, geralmente, após ocorrência de alterações físicas, cognitivas, doenças agudas ou crônicas, perda do cônjuge ou filho, perda de apoios sociais, pobreza, gerando dependência na realização das atividades da vida diária e locomoção. Trata-se de estudo clínico experimental controlado, realizado em uma casa de repouso em Vila Velha, ES, com o objetivo de avaliar a influencia da TAA na pressão arterial de idosos hipertensos institucionalizados. Participaram do estudo 25 idosos. As variáveis utilizadas no trabalho foram TAA (independente), variação de Pressão Arterial (dependente) e idade (controle). Foram realizadas sessões de TAA semanalmente com a duração de 1 hora e a pressão arterial foi aferida com uso de esfigmomanômetro antes e após a cada sessão durante 4 meses. Observou-se melhor controle dos níveis pressóricos além da promoção de momentos de alegria e relaxamento da amostra representativa do grupo de idosos institucionalizados.

Descritores: Idoso; Alternativas ao uso de animais; Pressão arterial.

ABSTRACT: The animal-assisted therapy (AAT) is used as a tool in the treatment of diseases, with several beneficial effects on psychiatric patients, hospitalized, elderly, among others. AAT aims to promote the well-being, emotional, physical, social and cognitive health, where the animal is the main therapeutical agent, working as a bridge between treatment and the patient.

Apresentação de resumo do trabalho na 22a Conferência Mundial de Promoção da Saúde. Saúde Soc (São Paulo). 2016;25:208.

1. Médica Veterinária, Mestre pela UFV, Coordenadora do Projeto Bicho Solidário, Docente da Universidade Vila Velha. Email: fetvieira@bol.com.br.

2. Médica, Mestre em Saúde Coletiva pela UFES, Docente da Universidade Vila Velha. Email: raciresampaio@gmail.com.

3. Fisioterapeuta, Mestre, Docente da Universidade Vila Velha. Email: valeriagon g@hotmail.com.

4. Agrônomo, Especialista em Estatística pela UFLA, Mestre pela UFV, Docente da Universidade Vila Velha. Email: romildoazevedo@ hotmail.com.

5. Estatístico, Doutor pela University of Southampton, Docente do Departamento de Estatística da UFJF. Email: mdtvieira@gmail. com.

6. Médico Veterinário, Mestre pela UFES, Docente da Universidade Vila Velha. Email: mrenan@uvv.br

7. Discente do Curso de Medicina da Universidade Vila Velha. Email: guadalupegcm@hotmail.com, daniele_vboj@hotmail.com, irineuln@gmail.com

Correspondência para: Irineu Vieira Lopes Neto. Email: irineuln@gmail.com. 
The institutionalization of the elderly usually occurs after the occurrence of physical, cognitive, acute or chronic illnesses, loss of spouse or child, loss of social support or poverty, creating dependency in performing activities of daily living and mobility. It is a controlled experimental clinical study, conducted in a nursing home in Vila Velha, ES, in order to evaluate the influence of the AAT in blood pressure of institutionalized elderly hypertensives. The study included 25 elderly. The variables used

\section{INTRODUÇÃO}

Terapia Assistida por Animais (TAA) consiste no uso de técnicas de utilização de animais auxiliando na recuperação de seres humanos com diversos problemas de saúde, estimulando tanto o aspecto físico quanto o emocional, com o objetivo de melhorar a qualidade de vida das pessoas e acelerar os processos de recuperação ${ }^{1}$. Padronizado pela organização americana Delta Society, trata-se de um processo terapêutico formal em âmbito mundial, com a utilização de procedimentos claros, apresentando muitos aspectos positivos e sendo uma estratégia adjuvante em diversos tratamentos ${ }^{2}$.

Especialistas têm considerado que a utilização do estímulo sensorial do tato com a presença e interação dos animais pode recuperar a auto-estima e a sensibilidade, além da reintegração à sociedade por meio da melhora do contato social ${ }^{3,4}$. A TAA traz consigo um aspecto importante de humanização, pois pode descontrair o clima tenso do ambiente hospitalar e clínico, melhorar as relações interpessoais e facilitar a comunicação entre pacientes e equipe de saúde. É indicada para auxiliar nas diversas situações clínicas, pois proporciona benefícios emocionais para os pacientes beneficiados 5 .

Efeitos positivos sobre os pacientes submetidos à TAA nos mais variados tipos de problemas de saúde têm sido observados, tais como demência senil ${ }^{6}$, mal de Alzheimer $^{7}$, esquizofrenia ${ }^{8}$, reabilitação de idosos ${ }^{9}$, e no tratamento de crianças e adolescentes com transtornos psicossociais ou hospitalizados ${ }^{5,10}$.

Além dos efeitos psicológicos, os animais também podem trazer benefícios fisiológicos para as pessoas. Alguns dos benefícios descritos são a redução dos níveis de triglicérides, colesterol, pressão sanguínea e estresse, diminuição da incidência de doenças cardiovasculares e maior facilidade de recuperação em caso de doenças ${ }^{11}$. Quando as pessoas interagem com os animais, falando com eles, acariciando-os ou manuseando-os, há diminuição da frequência cardíaca e pressão arterial, atingindo esta última, valores menores que os observados em pessoas saudáveis na situação de repouso ${ }^{12}$. Em outro estudo, em pacientes hospitalizados com insuficiência cardíaca, a TAA promoveu redução da pressão capilar pulmonar, redução dos níveis in the study were AAT (independent), changes in blood pressure (dependent) and age (control). AAT sessions were held weekly with duration of 1 hour and blood pressure was measured using a sphygmomanometer before and after each session for four months. There was better control of blood pressure levels besides a promotion of moments of joy and relaxation of a representative sample group of institutionalized elderly.

Keywords: Aged; Animal use alternatives; Arterial pressure.

hormonais de epinefrina e norepinefrina, além da redução da ansiedade $^{13}$.

A presença do animal em situações de estresse mental reduz a resposta ao mesmo reafirmando a influência dos fatores considerados tampão para a resposta fisiológica ao estresse em pacientes hipertensos tratados com medicamentos ${ }^{14}$.

Com o objetivo de avaliar a influência dos animais de companhia sobre a pressão arterial, um trabalho mensurou a pressão arterial de pessoas durante um teste aritmético diante da presença de seus animais de estimação e diante de algum amigo ou membro da família ${ }^{15}$. Constatou-se nessa pesquisa que mesmo diante da situação de estresse vivida durante o teste, na presença do animal, o aumento da pressão arterial foi pouco significativo quando comparado com o aumento observado na presença de pessoas. Tal fato foi justificado pela distração, relaxamento e redução do nível de estresse proporcionado pela presença do animal, ao passo que a presença do homem capaz de julgá-lo, influenciava grandes aumentos da pressão durante o teste.

Durante a pesquisa bibliográfica não foram localizados estudos em nossa região sobre o assunto, comprovando que não há pesquisas que avaliaram o efeito de TAA sobre a pressão arterial de pacientes idosos institucionalizados.

\section{OBJETIVO}

Esse estudo tem por objetivo avaliar a influência da TAA sobre a pressão arterial (PA) de idosos hipertensos institucionalizados, submetidos a tratamento medicamentoso para controle da PA.

\section{METODOLOGIA}

Trata-se de um estudo clínico experimental, controlado, aprovado pelo comitê de ética em pesquisa humana e pelo comitê de ética em pesquisa animal da Universidade Vila Velha (UVV).

A Pesquisa foi desenvolvida no período de agosto a novembro de 2010, na casa de repouso "Bem Viver", Vila 
Velha - ES. Foram objeto de estudo 25 idosos de ambos os sexos e com idade acima de 70 anos, hipertensos ${ }^{16}$, submetidos a tratamento medicamentoso para controle da PA, de acordo com prescrições médicas individuais, cujos responsáveis ou próprios pacientes manifestaram aquiescência em assinar o Termo de Consentimento para ser objeto de estudo, após esclarecimento sobre a pesquisa. Foram considerados como critérios de exclusão: aversão aos animais utilizados na terapia (cães e gatos) e contra indicações médicas de contato direto com esses animais.

Respeitados os critérios de inclusão e exclusão, os 25 idosos convidados foram divididos de forma randomizada em 2 grupos: participantes da TAA $(n=14)$ e não participantes $(n=11)$. Do grupo que teve contato com a terapia, ora chamado, grupo tratado, $71 \%$ eram do sexo feminino; e do grupo sem contato com os animais, ora chamado grupo controle, $45 \%$ eram do sexo feminino. Não se preocupou em controlar a variável sexo, uma vez que os estudos demonstram que essa característica não tem influência sobre os níveis pressóricos em pacientes desta faixa etária visto que, com a chegada da menopausa, a prevalência da pressão alta entre homens e mulheres tende a se aproximar ${ }^{17}$. Não houve conhecimento se havia a presença de animais de estimação no domicílio dos pesquisados antes da institucionalização e não foi considerado a história patológica pregressa de cada idoso individualmente.

As sessões de TAA foram realizadas semanalmente, com duração de 1 hora durante 4 meses. Durante esse período, os idosos realizavam caminhadas lentas e curtas conduzindo o animal, os acariciavam, conversavam com os animais, além de recordarem bons momentos do passado vividos ao lado do seu animal de estimação.

Para confirmar a homogeneidade, foram confrontadas as pressões arteriais dos idosos desses dois grupos antes do início da intervenção e não foi encontrada diferença significativa (Tabela 1).

Tabela 1. Comparativo entre grupos Controle e Tratado antes do início da terapia, julho 2010

\begin{tabular}{llcccc}
\hline Pressão & Grupo & n & Média & $\begin{array}{c}\text { Erro } \\
\text { padrão }\end{array}$ & p-valor* \\
\hline \multirow{2}{*}{ Sistólica } & Tratado & 12 & 123,33 & 1,88 & 0,36 \\
& Controle & 11 & 120,60 & 2,25 & \\
\hline \multirow{2}{*}{ Diastólica } & Tratado & 12 & 75,00 & 1,51 & 0,16 \\
& Controle & 11 & 71,36 & 1,94 & \\
\hline
\end{tabular}

Para avaliar a eficiência da TAA no controle pressórico dos idosos, foi aferida a pressão arterial dos idosos, por cinco alunos do curso de medicina, seguindo as recomendações das VI Diretrizes Brasileiras de Hipertensão Arterial ${ }^{16}$, em momentos distintos: antes do contato com os animais e depois, semanalmente, nos três últimos meses de terapia. No primeiro mês, só foi aferida a pressão após a terapia. A pressão foi avaliada com uso de esfigmomanômetro (Marca: BD) com paciente em posição sentada 1 hora após o termino da sessão de TAA. As aferições foram supervisionadas pelo mesmo professor em todos os momentos.

Para análise estatística descritiva foram utilizados gráficos, tabelas e as medidas de pressão arterial. Para análise estatística inferencial foi utilizado o teste $t$ para comparativos entre grupo controle e grupo tratado, e o teste $t$ pareado quando se objetivou comparar o grupo tratado antes e depois da terapia ou em meses distintos. O software utilizado foi o SPSS ${ }^{\circledR} 15.0$ e o nível de significância adotado foi de $5 \%$. Não foi necessário o uso de transformação de dados para normalização, pois os dados apresentaram condições de baixa assimetria e curtose satisfatória ao uso da abordagem paramétrica, além de não violarem a hipótese de normalidade pelo teste de Kolmogorov-Smirnov.

\section{RESULTADOS}

Nas Figuras 1 e 2, tem-se a representação gráfica para as pressões arteriais sistólica (Figura 1) e diastólica (Figura 2) analisadas no grupo tratado, onde se observa diferença significativa, a partir do segundo mês de observação, para a pressão diastólica, enquanto que, para a pressão sistólica, só houve diferença significativa no último mês observado. Sendo, respectivamente, V, W, X, Y e Z, os meses de julho a novembro.

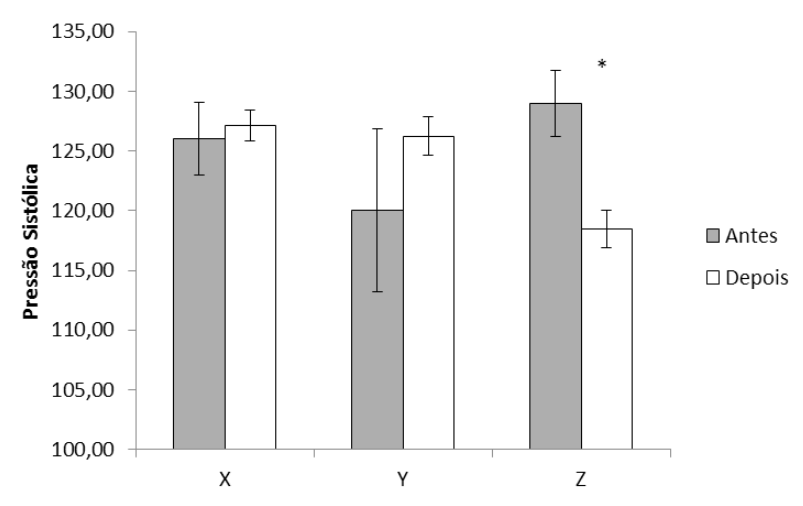

Figura 1. Médias e respectivos erros padrões para as pressões sistólica antes e após a terapia com animais nos três meses de tratamento $(\mathrm{X}, \mathrm{Y}$ e Z). Asterisco sobre as barras indica diferença significativa pelo teste $t$ pareado $(\mathrm{p}<0,05)$ 


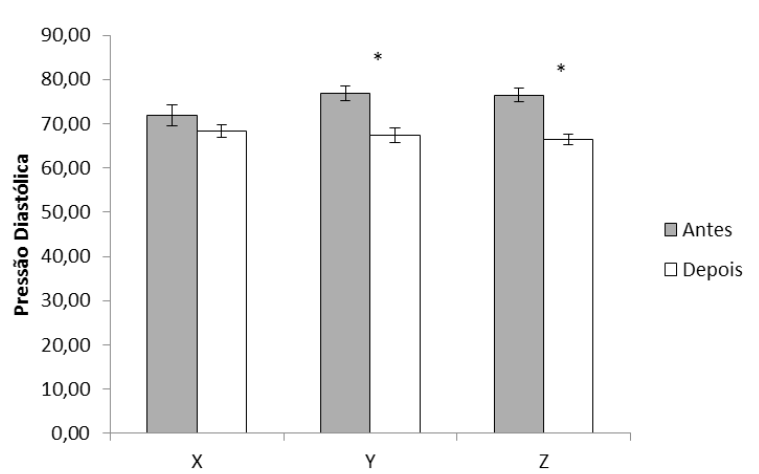

Figura 2. Médias e respectivos erros padrões para as pressões diastólica antes e após a terapia com animais nos três meses de tratamento $(\mathrm{X}, \mathrm{Y}$ e Z). Asteriscos sobre as barras indicam diferença significativa pelo teste $t$ pareado $(\mathrm{p}<0,05)$
No comparativo entre grupo controle e grupo submetido à terapia (tratado), observa-se, na Tabela 2, que as pressões dos idosos analisados começam a apresentar alguma diferença na pressão diastólica, já no mês de setembro, com um indicativo de significância, pois p-valor situa-se entre 5 e $10 \%$, confirmada com significância estatística em outubro, porém, com volta de indicativo de significância em novembro. Já a pressão sistólica não apresenta diferença significativa entre os dois grupos durante os meses de setembro e outubro e passa a apresentar indicativo de significância em novembro.

Na Figura 3, observa-se redução significativa da PA do grupo tratado na comparação de julho (antes do início da terapia) com novembro (4 meses após terapia) tanto da PA sistólica quanto da diastólica, após a terapia com animais, apesar da exiguidade da amostra.

Tabela 2. Comparativo entre grupos controle e tratado na aferição da PA

\begin{tabular}{|c|c|c|c|c|c|c|}
\hline Pressão & Mês & Grupo & $\mathbf{N}$ & Média & Erro padrão & p-valor \\
\hline \multirow{6}{*}{ Diastólica } & \multirow{2}{*}{$\mathrm{X}$} & Tratado & 14 & 67,80 & 1,11 & \multirow{2}{*}{0,058} \\
\hline & & Controle & 11 & 71,82 & 1,79 & \\
\hline & \multirow{2}{*}{$\mathrm{Y}$} & Tratado & 14 & 67,07 & 1,48 & \multirow{2}{*}{0,025} \\
\hline & & Controle & 11 & 72,45 & 1,69 & \\
\hline & \multirow{2}{*}{$\mathrm{Z}$} & Tratado & 14 & 66,37 & 1,13 & \multirow{2}{*}{0,051} \\
\hline & & Controle & 11 & 70,83 & 1,98 & \\
\hline \multirow{6}{*}{ Sistólica } & \multirow{2}{*}{$\mathrm{X}$} & Tratado & 14 & 126,64 & 0,98 & \multirow{2}{*}{0,332} \\
\hline & & Controle & 11 & 123,64 & 2,81 & \\
\hline & \multirow{2}{*}{$\mathrm{Y}$} & Tratado & 14 & 126,04 & 1,08 & \multirow{2}{*}{0,812} \\
\hline & & Controle & 11 & 125,41 & 2,39 & \\
\hline & \multirow{2}{*}{$\mathrm{Z}$} & Tratado & 14 & 118,54 & 1,55 & \multirow{2}{*}{0,052} \\
\hline & & Controle & 11 & 123,33 & 1,76 & \\
\hline
\end{tabular}

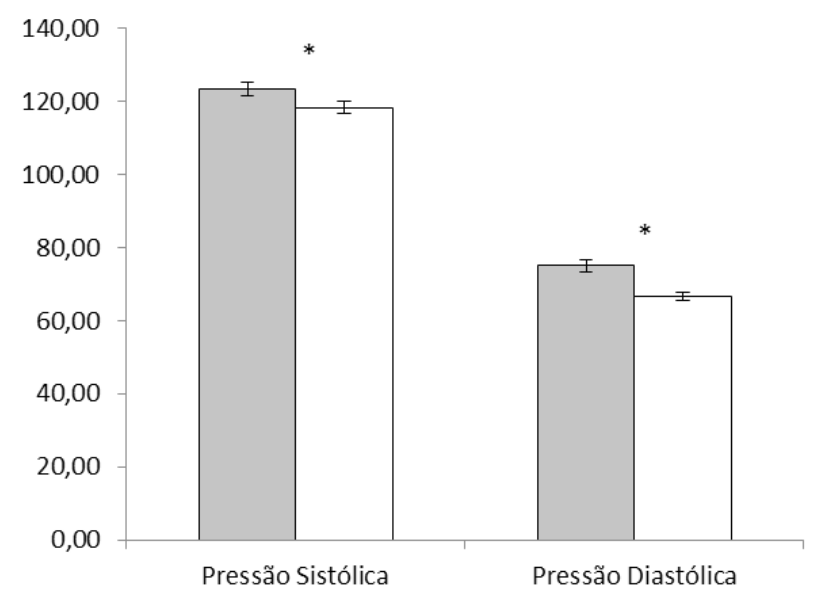

$\square \mathrm{V}$

Figura 3. Médias e respectivos erros padrões para as pressões aferidas em antes do início (V) e ao fim da terapia com animais (Z). Asteriscos para a mesma categoria de pressão, sobre as barras indicam diferença significativa entre os meses pelo teste $t$ pareado $(\mathrm{p}<0,05)$ 
Buscando avaliar se a queda foi progressiva com o prolongamento do contato com os animais e não somente com a presença do animal, comparou-se as médias de PA de agosto (somente 1 mês de contato) com novembro (3 meses de contato), quando, observou-se significância para ambas pressões (Figura 4), comprovando os benefícios da terapia continuada com os animais neste grupo populacional.

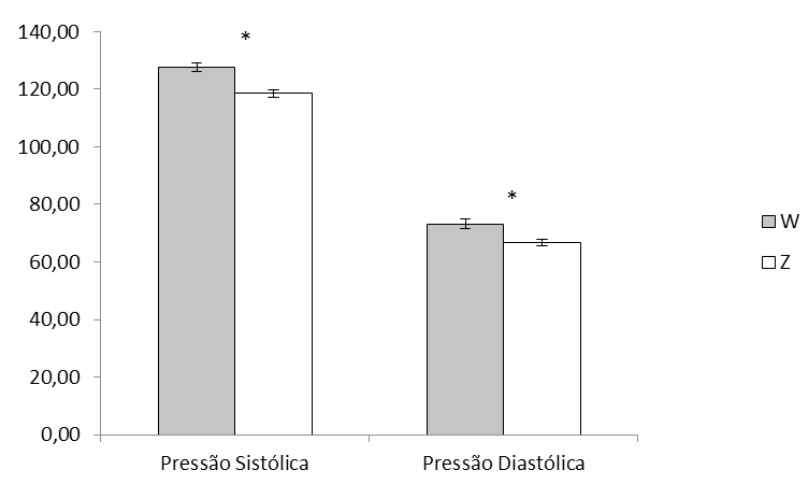

Figura 4. Médias e respectivos erros padrões para as pressões aferidas no início (W) e ao fim (Z) da terapia com animais. Asterisco para a mesma categoria de pressão, sobre as barras indica diferença significativa entre os meses pelo teste $t$ pareado $(\mathrm{p}<0,05)$

Além dos efeitos da TAA sobre a pressão arterial, observaram-se também outros benefícios nesse estudo. Observou-se mesmo que de forma subjetiva a transformação da dor, da tristeza e da solidão, a sensação de incapacidade e de limitações, sentimentos presentes em grande parte dos idosos institucionalizados, em alegria, prazer, confiança o que lhes proporcionou uma condição de vida mais agradável.

\section{DISCUSSÃO}

Quando analisados os resultados, foram encontradas diferenças significativas entre a pressão arterial, tanto sistólica quanto diastólica, entre os idosos que tiveram contato com animais durante as sessões de TAA, reforçando a importância do vínculo homem-animal.

A redução da pressão arterial foi observada em idosos em outro estudo com uma combinação de atividades e exercícios físicos que incluíram a caminhada, atividades recreativas e alongamento ${ }^{18}$, além da presença dos animais que estimulava a realização de caminhadas lentas de pequenas distâncias os conduzindo a um agradável passeio e dos exercícios com as mãos no momento em que acariciavam os mesmos.
Notou-se de forma evidente o efeito positivo no controle da PA dos idosos institucionalizados, estando de acordo com dados a literatura, que constatou que quando as pessoas interagem com os animais, falando com eles, acariciando-os ou manuseando-os, há diminuição da frequência cardíaca e $\mathrm{PA}^{12}$. Além disto, a TAA proporcionou aos idosos um momento de alegria, relaxamento e uma maior socialização entre pacientes e profissionais (médico, fisioterapeuta, médico veterinário e adestrador de animais, além de voluntários condutores de animais).

A sensação de felicidade com a chegada dos animais era evidente com a presença de sorrisos notados em cada idoso. As sessões de TAA promoveram momentos de tranquilidade e descontração entre os idosos e toda a equipe de saúde participante. Estímulos cognitivos foram executados ao recordarem situações vivenciadas no passado com seus animaizinhos de estimação ${ }^{9}$. É provável que este clima agradável, gerado pela presença dos animais coterapeutas, tenha também sido responsável pela redução da ansiedade e tenha funcionado como um tampão para a resposta fisiológica a condições estressantes do dia-a-dia que poderiam culminar com aumentos da PA, estando de acordo com dados observados em outros estudos ${ }^{13,14}$. Cabem ressaltar que o estresse e a ansiedade são considerados, atualmente, importantes fatores predisponentes para a ocorrência de doenças cardiovasculares ${ }^{19}$.

\section{CONCLUSÃO}

Mediante os resultados, concluímos que a TAA foi capaz de atuar beneficamente em funções fisiológicas melhorando o padrão cardiovascular e diminuindo a PA. Embora os resultados promovam evidências da redução da PA em idosos hipertensos submetidos a tratamento medicamentoso associada a TAA, em virtude da existência limitada de estudos nesta área e do número reduzido de nossa amostra, este trabalho abre a expectativa para a realização de outros semelhantes envolvendo um número maior de pacientes com o objetivo de avaliar a influência da TAA sobre a pressão arterial além de estabelecer o mecanismo exato envolvido, bem como avaliar a influência da TAA sobre outros fatores fisiológicos como a função endócrina e sobre o alívio do estresse.

Como as doenças cardiovasculares representam uma importante causa de morte em todo o mundo e a hipertensão apresenta elevada incidência entre idosos, o estabelecimento do real efeito da TAA sobre o controle da pressão arterial trará uma excelente medida adjuvante e não medicamentosa ao tratamento destes pacientes com baixo custo. 


\section{REFERÊNCIAS}

1. Dotti J. O que é a A/TAA? In: Dotti, J. Terapia \& animais. São Paulo: PC Editorial; 2005. p.29-37.

2. Delta Society. Atividade e terapia assistida por animais (A/ TAA). 2005 [acesso 19 mar. 2005]. Disponível em: http:// www.projetocao.com.br/main.htm.

3. Martins MF. Zooterapia ou terapia assistida por animais (TAA). Rev Nosso Clínico. 2004;40:24-6.

4. San Joaquín MPZ. Terapia assistida por animales de conpañia. Bienestar para el ser humano. Temas Hoy. 2002;143-9.

5. Vaccari AMH, Almeida FA. A importância da visita de animais de estimação na recuperação de crianças hospitalizadas. Einstein. 2007;5(2):111-6. Disponível em: http://patastherapeutas.org/wp-content/uploads/2015/07/ Animais-de-estimaa\%CC \% 81\%E2\%88\%86o-ehospitalizaa\%CC\%81\%E2\%88\%86o.pdf.

6. Kanamori M, Suzuki M, Yamamoto K, Kanda M, Matsui Y, Kojima E, et al. A day care program and evaluation of animal-assisted therapy (AAT) for the elderly with senile dementia. Am J Alzheimers Dis Other Demen. 2001;16(4):234-9. doi: 10.1177/153331750101600409.

7. Edwards NE, Beck AM. Animal-assisted therapy and nutrition in Alzheimer's disease. West J Nurs Res. 2002;24(6):697-712. doi: 10.1177/019394502320555430.

8. Kovacs Z, Kis R, Rozsa S, Rozsa L. Animal-assisted therapy for middle-aged schizophrenic patients living in a social institution. A pilot study. Clin Rehabil. 2004;18(5):483-6. doi: 10.1191/0269215504cr765oa.

9. Pecelin A, Furlan LA, Berbel AM, Lanuez FV. Influência da fisioterapia assistida por animais em relação à cognição de idosos - estudo de atualização. ConScientiae Saúde (São Paulo). 2007;6(2):235-40. Disponível em: http:// patastherapeutas.org/wp-content/uploads/2015/07/ Influencia-da-terapia-assistida-por-animais-com-idosos. pdf.

10. Bardill N. Animal assisted therapy with hospitalized adolescents [thesis]. Florida: College of Nursing, University of Florida; 1994.
11. Jofre ML. Visita terapéutica de mascotas en hospitales. Rev Chilena Infectol. 2005;22(3):257-63. http://dx.doi. org/10.4067/S0716-10182005000300007.

12. Lynch JJ, Thomas SA, Long JM, Malinow KL, Chickadonz G, Katcher AH. Humam speech and blood pressure. J Nerv Ment Dis. 1980;168(9):526-34.

13. Cole KM, Gawlinski A, Steers N, Kotlerman. Animalassisted therapy in patients hospitalized with heart failure. Am J Crit Care. 2007;16:575-85. Available from: http:// ajcc.aacnjournals.org/content/16/6/575.long.

14. Allen K, Shykoff BE, Izzo JL. Pet ownership, but not ACE inhibitor therapy, blunts home blood pressure responses to mental stress. Hypertension. 2001;38:815-20. Available from: http://hyper.ahajournals.org/content/38/4/815.long.

15. Allen K. Are Pets a Healthy Pleasure? The influence of pets on blood pressure. Curr Direct Psychol Scie. 2003;12:236-9. doi: 10.1046/j.0963-7214.2003.01269.x.

16. Sociedade Brasileira de Cardiologia; Sociedade Brasileira de Hipertensão; Sociedade Brasileira de Nefrologia. VI Diretrizes Brasileiras de Hipertensão. Arq Bras Cardiol. 2010;95(1):1-51. Disponível em: http://publicacoes.cardiol. br/consenso/2010/Diretriz_hipertensao_associados.pdf.

17. Silva JLL, Souza SL. Fatores de risco para hipertensão arterial sistêmica versus estilo de vida docente. Rev Eletrônica Enfermagem. 2004;6(3) [acesso em 2011 jul 27]. Disponível em: https://www.revistas.ufg.br/fen/article/ view/838/989.

18. Ishikawa $\mathrm{K}$, Ohta $\mathrm{T}$, Zhang J, Hashimoto $\mathrm{S}$, Tanaka $\mathrm{H}$. Influence of age and gender on exercise training-induced blood pressure reduction in systemic hypertension. Am J Cardiol. 1999;84:192-6. http://dx.doi.org/10.1016/S00029149(99)00233-7.

19. Siegel JM. Stressful life events and use of physician services among the elderly: the moderating role of pet ownership. J Pers Soc Psychol. 1990;58:1081-6. http:// dx.doi.org/10.1037/0022-3514.58.6.1081. 